\title{
Integrating molecular mechanisms into quantitative genetics to understand consistent individual differences in behavior
}

\author{
Alison M. Bell ${ }^{\mathrm{a}, *}$ \\ ${ }^{a}$ School of Integrative Biology, Carl R. Woese Institute for Genomic Biology, Neuroscience \\ Program and Program in Ecology, Evolution and Conservation.
}

Ned A. Dochtermann ${ }^{\mathrm{b}}$

${ }^{\mathrm{b}}$ Department of Biological Sciences, Dept. 2715, North Dakota State University, PO Box 6050, Fargo, ND 58108-6050

$2{ }^{*}$ Corresponding author, 505 S. Goodwin Ave., University of Illinois at Urbana-Champaign, 3 Urbana, IL 61801, USA. 217-265-5469 (phone), 217-244-4565 (fax), alisonmb@ life.illinois.edu 


\section{Abstract}

7 It is now well appreciated that individual animals behave differently from one another and that

8 individual differences in behaviors — personality differences — are maintained through time and

9 across situations. Quantitative genetics has emerged as a conceptual basis for understanding the

10 key ingredients of personality: (co)variation and plasticity. However, the results from

11 quantitative genetic analyses are often divorced from underlying molecular or other proximate

12 mechanisms. This disconnect has the potential to impede an integrated understanding of behavior

13 and is a disconnect present throughout evolutionary ecology. Here we discuss some of the main

14 conceptual connections between personality and quantitative genetics, the relationship of both

15 with genomic tools, and areas that require integration. With its consideration of both trait

16 variation and plasticity, the study of animal personality offers new opportunities to incorporate

17 molecular mechanisms into both the trait partitioning and reaction norm frameworks provided by

18 quantitative genetics.

19

20

21 


\section{Introduction}

23 A thriving area of research in the study of animal behavior involves understanding

24 consistent individual differences ('animal personality'). There is growing appreciation that

25 individual animals within natural populations behave differently from one another, and that they

26 retain these behavioral differences through time and across situations. Consistent individual

27 differences in behavior are interesting to animal behaviorists for at least three reasons. First, we

28 want to know why individuals are different from one another; in other words, why is there

29 variation? Second, we want to understand why behavioral traits are correlated in particular

30 configurations (i.e. as components of behavioral syndromes). Why, for example, are boldness

31 and aggressiveness correlated in some populations but not others? Third, we want to know why

32 individuals have a behavioral type that they maintain over time - what limits behavioral

33 plasticity? The key features of personality — among individual (co)variation and within

34 individual consistency — are what distinguishes the study of personality from simply the study of

35 behavior.

36 Interest regarding animal personalities increasingly integrates research across levels of

37 analysis and combines different methodological approaches. In particular there is growing

38 interest in evolutionary processes that can generate consistent individual differences and

39 widespread appreciation that understanding the proximate mechanisms underlying personality

40 can shed light on its causation and evolution.

41

42 Quantitative genetics provides a framework for understanding personality

43 Quantitative genetics provides a strong conceptual basis for considering the key

44 ingredients of personality: (co)variation and plasticity [1]. In particular, quantitative genetics 
45 provides a statistical framework for partitioning trait variation, covariation within and among

46 individuals, and can estimate population and individual level behavioral plasticity [1]. For

47 example, mixed effects statistical models can estimate reaction norms — phenotypic plasticity-

48 using the phenotypic equation [2]:

$$
\left.y_{i j}=\left(\beta_{0}+i n d_{0 j}\right)+\left(\beta_{1}+i n d_{1 j}\right) x_{i j}+e_{0 i j} \quad \text { (equation } 1\right)
$$

50 where $y_{i j}$ is the behavioral response of individual $j$ at instance $i[3]$. The different parts of the

51 equation correspond to population level average behavioral responses $\left(\beta_{0}\right.$, assuming centering of

52 predictor variables), the individual's deviation from this average ( $i n d_{0 j}$, i.e. personality

53 variation), and residual variation for that individual at that particular instance $\left(e_{0 i j}\right)$. Plasticity

54 enters via $x_{i j}$, which is the environment experienced by that individual at that instance. If the

55 population as a whole shows behavioral plasticity, this can be estimated $\left(\beta_{1}\right)$ and individual level

56 plasticity can also be estimated $\left(i n d_{1 j}\right)$. Individual average behavior as it differs from the

57 population mean $\left(i n d_{0 j}\right)$ and individual plasticity as it differs from population level plasticity

$58 \quad\left(\right.$ ind $\left._{1 j}\right)$ might also be correlated such that personality and plasticity are correlated (for further

59 details and assumptions see [3]). The emergence of quantitative genetics as an organizational

60 basis for the field of personality research has allowed the rapid translation of statistical methods

61 to the field and highlighted evolutionary questions of potential importance (e.g. [4]).

\section{The problem: How to incorporate molecular mechanisms?}

However, while quantitative genetics provides a conceptual basis for understanding

65 consistent individual differences, the results from quantitative genetic analyses are often

66 statistical abstractions, divorced from underlying molecular or other proximate mechanisms.

67 Indeed, the prevailing approaches for studying the genetic basis of traits tend to focus on either 
68 the quantitative genetic or molecular side of the equation, and the two approaches require very

69 different tools. Studies of quantitative genetics and molecular mechanisms are also often carried

70 out in isolation of each other and their results are difficult to integrate. For example, one study

71 might compute a genetic correlation between two behaviors [5], while another study in the same

72 species finds correlated patterns of genome-wide expression associated with these two behaviors

73 [6,7]. Similarly, one study might estimate the slope of a behavioral reaction norm across

74 environments, while another shows that gene expression changes across environments.

75 Unfortunately the results from these approaches are not immediately comparable.

76 The gap between the statistical abstractions of quantitative genetics and the actual genetic

77 or molecular mechanisms underlying them can be a problem because different mechanisms can

78 produce the same outcome, and/or have different implications for evolution. For example, a

79 relatively low estimate of heritability could reflect either low genetic variation or high non-

80 genetic variation. Similarly, a genetic correlation between two behaviors could reflect either

81 pleiotropy (when the same genes influence the two traits), physical linkage between loci or

82 selection-induced linkage disequilibrium (when the traits are influenced by different, co-selected

83 genes) [4,5,8]. Finally, phenotypic plasticity could reflect allelic sensitivity (different genes

84 underlie the trait in different environments) or gene regulation (the same gene influences the trait

85 in different environments, but the gene is regulated differently in the two environments [9]).

86 Bridging this gap is an important priority for all of biology and emerging interest in personality

87 offers an opportunity for combining molecular mechanisms and quantitative genetic approaches.

88 Arguably, the advancement of personality research — with its concern for both variation among-

89 individuals and for plasticity—necessitates this synthesis. 
One strategy for bridging the divide between molecular mechanisms and traditional

91 quantitative genetics is to identify the specific genetic variants that contribute to additive genetic

92 variation and thus to phenotypic variation. Identification of such genetic variants can be

93 achieved via quantitative trait loci (QTL) studies and genome-wide association studies (GWAS).

94 While such approaches are currently limited to studying traits underlain by genes of large effect

95 [10], finding such genes can be informative for understanding the proximate basis of traits, and

96 can be used to address longstanding questions about the genetic basis of adaptation [11].

97 Identification of such genes also allows better understanding of the evolution of behavior and

98 whether particular responses might be deeply conserved or novel. Finally, intensive public

99 investment in GWAS was driven by concerns regarding human health, for which understanding

100 how particular genes affect behavior can be highly relevant [12].

101 A number of recent papers have identified QTL associated with behavioral variation in

102 natural populations, e.g. burrowing behavior in mice [13], schooling behavior in sticklebacks

103 [14], aggressive behavior in Drosophila [15] and feeding behavior in C. elegans [16].

104 Importantly, such information from QTL studies can be integrated into the statistical framework

105 provided by traditional quantitative genetics. For example, the proportion of phenotypic variation

106 attributable to QTL variation can be framed in terms of heritability due to that QTL (but see

107 [17]). Further, the phenotypic equation can be modified to include information about the

108 influence of particular loci (e.g. QTL, expanding from [18]):

$$
y_{i j m}=\left(\beta_{0}+i n d_{0 j}+G_{0 m}\right)+\left(\beta_{1}+i n d_{1 j}\right) x_{i j}+e_{0 i j} \quad \text { (equation 2) }
$$

110 where the individual's phenotype is now also determined by a known loci $(m$, e.g. a particular

111 QTL) which has an estimable average contribution to the phenotype $\left(G_{0 m}\right.$; as above this

112 represents a deviation from the population level average, $\beta_{0}$ ). Within this framework, the 
113 contribution of variation at specific loci and epistatic interactions among them can thereby be

114 estimated. Moreover, this framework can also be extended to include not only directly estimable

115 genetic influences on behavior but also inferred genetic influences when pedigree information is

116 known and relatedness included (i.e. application of the "animal model", [19]). However, it is

117 likely to be challenging to apply this general framework to natural variation in behavioral traits

118 with fitness consequences, because they are typically underlain by hundreds of genes of small

119 effect and influenced by both genetic variation and the environment [20].

120 In principle, equation 2 can incorporate information about particular genes that might

121 contribute to individual variation in plasticity, via the same manner by which individual

122 plasticity was included:

123

$$
\left.y_{i j m}=\left(\beta_{0}+i n d_{0 j}+G_{0 m}\right)+\left(\beta_{1}+i n d_{1 j}+G_{1 m}\right) x_{i j}+e_{0 i j} \quad \text { (equation } 3\right)
$$

124 where the individual's known genotype $(m)$ now has an estimable plastic effect on the behavior

125 of the individual $\left(G_{1 m}\right)$ based on the environment currently experienced $\left(x_{i j}\right)$. However it is

126 likely to be challenging to empirically obtain these estimates.

127 An alternative to QTL and GWAS that comes closer to capturing molecular mechanisms

128 associated with plasticity involves measuring genome-wide gene expression (e.g. [21-23]). The

129 appeal of measuring genome-wide expression, as opposed to focusing on fixed genetic variation,

130 is that it can reveal which genes in the genome are responsive to the environment, and therefore

131 likely to be related to phenotypic plasticity. For example, recent genome-wide transcription

132 studies have revealed that roughly $\sim 10 \%$ of all of the genes in the genome are differentially

133 expressed in response to a mating opportunity [24-29], predation risk [7,30,31], or a territorial

134 challenge $[6,32,33]$. Recent evidence suggests that transcriptional responses can be the result of a

135 conserved genomic response to social challenges [34]. Genome-wide gene expression studies 
136 have also detected differences in brain gene expression between behavioral types, e.g. scouts vs

137 nonscout honey bees [35] and alternative mating types [29,36,37]. A few particularly exciting

138 recent studies have both compared gene expression between behavioral types and changes in

139 gene expression in response to the environment $[29,38]$. This strategy can inform our

140 understanding of the evolution of plasticity by identifying potential targets of selection on

141 plasticity [39]. However, studies measuring gene expression in regards to behavior largely occur

142 in isolation of studies aimed at quantifying repeatability, heritability and genetic correlations -

143 the traditional province of quantitative genetics (but see eQTL [40]). Unfortunately, how results

144 from genome-wide gene expression studies translate to the conceptual framework of quantitative

145 genetics remains to be resolved. This is not a trivial problem and there are several specific (e.g.

146 Box 1) and general questions that must be addressed. It is, however, an essential problem as our

147 theoretical understanding of evolution is in large part tied to quantitative genetic theory.

149 Conclusions: Personality requires integration

150 Understanding personality—consistent individual differences—requires consideration of

151 both variation and plasticity. The study of personality has prompted questions about how

152 processes that can generate variation can coexist alongside processes that allow within-individual

153 plasticity, and indeed, how those processes are related to each other [41]. The study of

154 personality therefore offers an opportunity for integration and improved understanding of both

155 individual differences and behavioral plasticity. The study of personality also prompts a

156 comprehensive synthesis of molecular mechanisms into both the variance partitioning and

157 reaction norm frameworks provided by quantitative genetics. This integration of stability and

158 plasticity alongside the integration of molecular mechanisms and quantitative genetics represents 
159 the next frontier for personality research and will similarly advance not only the study of animal

160 behavior but evolutionary ecology as a whole.

162 Box 1: Sample challenges for integrating molecular mechanisms into quantitative genetics, 163 as applied to personality

164 - Can genome-wide gene expression data be used to link the $\mathbf{P}$ and $\mathbf{G}$ matrix?

165 - How to relate pleiotropy via a genetic correlation with patterns of gene expression?

166 - How to relate $\mathrm{I} \times \mathrm{E}$ or $\mathrm{G} \times \mathrm{E}$ reaction norms estimated within a quantitative genetic

167 framework with gene expression measured in different environments?

168 - Is there a way to detect permanent environment, among-individual, within-individual and

169 residual effects in gene expression data?

170 - Do differences in brain gene expression between behavioral types reflect additive genetic

171 variation, among-individual variation or within-individual variation?

172

173 Acknowledgements

174 Work in AMB's lab has been supported by grants from the National Science Foundation (IOS

175 1121980) and the National Institutes of Health (R01 GM082937).

177 References cited

178

179

180

181

182

183

184
1. Dingemanse NJ, Dochtermann NA: Individual behaviour: behavioural ecology meets quantitative genetics. In Quantitative genetics in the wild. Edited by A. Charmantier DG, and L. E. B. Kruuk: Oxford University Press; 2014.

2. Nussey DH, Wilson AJ, Brommer JE: The evolutionary ecology of individual phenotypic plasticity in wild populations. Journal of Evolutionary Biology 2007, 20:831-844. 
3. **Dingemanse NJ, Dochtermann NA: Quantifying individual variation in behaviour: mixed-effect modelling approaches. Journal of Animal Ecology 2013, 82:39-54. Provides a statistical framework for integrating individual variation in behavior and plasticity into quantitative genetic theory.

4. Dochtermann NA, Dingemanse NJ: Behavioral syndromes as evolutionary constraints. Behavioral Ecology 2013, 24:806-811.

5. Bell AM: Differences between individuals and populations of threespined stickleback. Journal of Evolutionary Biology 2005, 18:464-473.

6. Sanogo YO, Band, M.A., Blatti, C., Sinha, S. \& Bell, A.M. : Transcriptional regulation of brain gene expression in response to a territorial intrusion. Proceedings of the Royal Society B-Biological Sciences 2012, 279:4929-4938.

7. Sanogo YO, Hankison S, Band M, Obregon A, Bell AM: Brain transcriptomic response of threespine sticklebacks to cues of a predator. Brain Behavior and Evolution 2011, 77:270-285.

8. Sih A, Bell AM, Johnson JC, Ziemba R: Behavioral syndromes: an integrative overview. Quarterly Review of Biology 2004, 79:241-277.

9. Schlichting $\mathrm{CD}$, Pigliucci M: Gene regulation, quantitative genetics and the evolution of reaction norms. Evolutionary Ecology 1995, 9:154-168.

10. Rockman MV: The QTN program and the alleles that matter for evolution: all that's gold does not glitter. Evolution 2012, 66:1-17.

11. *Rausher MD, Delph LF: When does understanding phenotypic evolution require identification of the underlying genes? Evolution In press:n/a-n/a. Articulates when and why "knowing the genes" matters.

12. Visscher Peter M, Brown Matthew A, McCarthy Mark I, Yang J: Five Years of GWAS Discovery. The American Journal of Human Genetics 2012, 90:7-24.

13. Weber JN, Peterson BK, Hoekstra HE: Discrete genetic modules are responsible for complex burrow evolution in Peromyscus mice. Nature 2013, 493:402-405.

14. Greenwood AK, Wark AR, Yoshida K, Peichel CL: Genetic and neural modularity underlie the evolution of schooling behavior in threespine sticklebacks. Current Biology 2013, 23:1884-1888.

15. Edwards AC, Mackay TF: Quantitative trait loci for aggressive behavior in Drosophila melanogaster. Genetics 2009, 182:889-897.

16. Bendesky A, Tsunozaki M, Rockman MV, Kruglyak L, Bargmann CI: Catecholamine receptor polymorphisms affect decision-making in C. elegans. Nature 2011, 472:313318.

17. Bogdan M, Doerge RW: Biased estimators of quantitative trait locus heritability and location in interval mapping. Heredity 2005, 95:476-484.

18. Walsh B: Quantitative genetics in the age of genomics. Theoretical Population Biology 2001, 59:175-184.

19. Wilson AJ, Reale D, Clements MN, Morrissey MM, Postma E, Walling CA, Kruuk LEB, Nussey DH: An ecologist's guide to the animal model. Journal of Animal Ecology 2010, 79:13-26.

20. Mackay TFC: The genetic architecture of behavior: Lessons from Drosophila. Genetica 2009, 136:295-302.

21. Harris RM, Hofmann HA: Neurogenomics of behavioral plasticity. Advances Experimental Medicine Biology 2014, 781:149-168. 
22. *Cardoso SD, Teles MC, Oliveira RF: Neurogenomic mechanisms of social plasticity. $J$ Experimental Biology 2015, 218:140-149.

Reviews molecular mechanisms likely to contribute to short-and long-term behavioral plasticity.

23. Bell AM, Aubin-Horth N: What whole genome expression data can tell us about the ecology and evolution of personality in animals. Philosophical Transactions of the Royal Society 2010.

24. McGraw LA, Clark AG, Wolfner MF: Post-mating gene expression profiles of female Drosophila melanogaster in response to time and to four male accessory gland proteins. Genetics 2008, 179:1395-1408.

25. Mack PD, Kapelnikov A, Heifetz Y, Bender M: Mating-responsive genes in reproductive tissues of female Drosophila melanogaster. Proceedings of the National Academy of Sciences of the United States of America 2006, 103:10358-10363.

26. Lawniczak MKN, Begun DJ: A genome-wide analysis of courting and mating responses in Drosophila melanogaster females. Genome 2004, 47:900-910.

27. Cummings ME, Larkins-Ford J, Reilly CRL, Wong RY, Ramsey M, Hofmann HA: Sexual and social stimuli elicit rapid and contrasting genomic responses. Proceedings of the Royal Society B-Biological Sciences 2008, 275:393-402.

28. Carney GE: A rapid genome-wide response to Drosophila melanogaster social interactions. Bmc Genomics 2007, 8:-.

29. *Fraser BA, Janowitz I, Thairu M, Travis J, Hughes KA: Phenotypic and genomic plasticity of alternative male reproductive tactics in sailfin mollies. Proceedings of the Royal Society B-Biological Sciences 2014, 281:20132310.

Uses brain gene expression profiling to identify genes associated with behavioral variation and behavioral plasticity.

30. Lavergne SG, McGowan PO, Krebs CJ, Boonstra R: Impact of high predation risk on genome-wide hippocampal gene expression in snowshoe hares. Oecologia 2014, 176:613-624.

31. Jansen M, Vergauwen L, Vandenbrouck T, Knapen D, Dom N, Spanier KI, Cielen A, De Meester L: Gene expression profiling of three different stressors in the water flea Daphnia magna. Ecotoxicology 2013, 22:900-914.

32. Alaux C, Sinha S, Hasadsri L, Hunt GJ, Guzman-Novoa E, DeGrandi-Hoffman G, UribeRubio JL, Southey BR, Rodriguez-Zas S, Robinson GE: Honey bee aggression supports a link between gene regulation and behavioral evolution. Proceedings of the National Academy of Sciences of the United States of America 2009, 106:15400-15405.

33. *Rittschof CC, Robinson GE: Manipulation of colony environment modulates honey bee aggression and brain gene expression. Genes Brain and Behavior 2013, 12:802-811. Shows that some of the genes that are responsive to a social challenge are deeply conserved.

34. Rittschof CC, Bukhari SA, Sloofman LG, Troy JM, Caetano-Anollés D, Cash-Ahmed A, Kent M, Lu X, Sanogo YO, Weisner PA, et al.: Neuromolecular responses to social challenge: Common mechanisms across mouse, stickleback fish, and honey bee. Proceedings of the National Academy of Sciences 2014, 111:17929-17934.

35. Liang ZS, Nguyen T, Mattila HR, Rodriguez-Zas SL, Seeley TD, Robinson GE: Molecular determinants of scouting behavior in honey bees. Science 2012, 335:1225-1228. 
36. Stiver KA, Harris RM, Townsend JP, Hofmann HA, Alonzo SH: Neural gene expression profiles and androgen levels underlie alternative reproductive tactics in the ocellated wrasse, Symphodus ocellatus. Ethology 2015, 121:152-167.

37. Aubin-Horth N, Landry CR, Letcher BH, Hofmann HA: Alternative life histories shape brain gene expression profiles in males of the same population. Proceedings of the Royal Society B-Biological Sciences 2005, 272:1655-1662.

38. Alaux C, Sinha S, Hasadsri L, Hunt GJ, Guzmán-Novoa E, DeGrandi-Hoffman G, UribeRubio JL, B.R S, Rodriguez-Zas S, Robinson GE: Honey bee aggression supports a link between gene regulation and behavioral evolution. Proceedings of the National Academy of Sciences of the United States of America 2009, 106:15400-15405.

39. West-Eberhard MJ: Developmental Plasticity and Evolution. Oxford: Oxford University Press; 2003.

40. Gilad Y, Rifkin SA, Pritchard JK: Revealing the architecture of gene regulation: the promise of eQTL studies. Trends in Genetics 2008, 24:408-415.

41. Stamps JA, Groothuis TGG: The development of animal personality: relevance, concepts and perspectives. Biological Reviews 2010, 85:301-325. 